\title{
Bildung im Alter im Kontext des dritten und vierten Lebensalters - Narrationen und Narrative
}

\author{
Ines Himmelsbach
}

Online publiziert: 12. März 2015

(C) Die Autor(en) 2015. Dieser Artikel ist auf Springerlink.com mit Open Access verfügbar.

Zusammenfassung Der Beitrag lotet die Chancen der Nutzung des gerontologischen Datensatzes der Interdisziplinären Längsschnittstudie des Erwachsenenalters (ILSE) für bildungsbiografische Analysen aus. Das Altern selbst zu lernen erscheint dabei als zentrale Analysekategorie. Der Beitrag nimmt Stellung zu aktuellen Debatten um Bildung im Alter und problematisiert deren sozialpolitisch-aktivierende und programmatisch ausgerichtete Fokussierung. In einem zweiten Schritt wird erläutert, inwiefern für die offene Frage, was Altern lernen im Prozess des Alterns bedeutet, Anschlüsse an die Bildungsbiografieforschung hilfreich sein können. Um Erzählungen über den Prozess des Alterns analysieren zu können, bedarf es des Rückgriffs auf einen Datensatz, der diesen Prozess beobachten kann. Die ILSEStudie bietet mit ihren Biographischen Explorationen über einen Zeitraum von 20 Jahren außerordentliche Möglichkeiten. Exemplarisch wird ein Fall aus dem ILSE-Datensatz ausgewählt, um einen möglichen Analyseweg zu skizzieren. Als Heuristik für einen relevanten Kontext des Alterns werden die von Lessenich (2014) portraitierten Narrative des Ruhestands, des Unruhestands und des Produktiven Alters mit zwei Narrationen aus den Jahren 1994 und 2005 relationiert.

\begin{abstract}
The article discusses the chances of using a gerontological data base (Interdisciplinary Longitudinal Study of Adulthood, German Acronym: ILSE) for analyses in educational biography research: Learning to age tends to be the central category of this analyses. The article comments on the current debate of aging and education and expounds the problems of politically driven activation of elders and the programmatic turn in the discussion. In a second step the article elaborates on what terms it is helpful to connect this research to current debates of biographical research on education. In order to be able to analyse narrations along the process of aging, it is necessary to resort on a dataset which is able to observe this pro-
\end{abstract}

Prof. Dr. I. Himmelsbach $(\bowtie)$

Katholische Hochschule Freiburg, Karlstr. 63, 79104 Freiburg, Deutschland

E-Mail: ines.himmelsbach@kh-freiburg.de 
cess. The database of ILSE covering "biographical explorations" over a period of 20 years provides extraordinary opportunities. In this article, a case study of the ILSE dataset is selected in order to provide a potential way of further analyses. Narratives of Ageing (retirement, active retirement, productive aging) are selected as a heuristic for a relevant context of aging and those are related to two narratives of one individual at two points in previous time (1994 and 2005).

\section{Startpunkt - Standpunkte zu Bildung und Alter}

Bildung im Alter umfasst einen Zeitraum von bis zu fünf Jahrzehnten. Adressaten von Altersbildung können ältere Arbeitnehmer wie Hochaltrige am Ende ihres Lebens sein. Weiterhin ist Bildung im Alter als Phänomen zu betrachten, das ,lifewide“ (Alheit und Dausien 2002) zu beobachten ist, vom informellen Lernen im Alltag, dem Übergang in den Ruhestand, den non-formalen mit Zertifikaten ausgestatteten Lernprojekten zur Vorbereitung auf ein ehrenamtliches Engagement bis hin zu formalisierten Lernprozessen an Universitäten des dritten Lebensalters. Was allerdings wenig beachtet wird in der erziehungswissenschaftlichen Betrachtung des Phänomens Alter und Bildung, ist die Veränderung des Individuums in diesem Zeitraum. Eine Veränderung von individuellen Bildungsprozessen und gesellschaftlichen Kontexten in bis zu fünf Dekaden kann kaum bestritten werden. Um diese sich wandelnden und vielfältigen Lernanlässe und Bildungsprozesse theoretisch zu umreißen, wird ein weiter Bildungsbegriff benötigt, der es ermöglicht, das Phänomen Bildung im Alter in erzählten Biografien empirisch zu fassen. Folgt man Kade und Nolda (2014b), so

besteht die zunehmend selbstverständlich gewordene Erwartung an jeden Einzelnen, sein Leben selbstverantwortlich zu gestalten und sich, orientiert an neuen gesellschaftlichen Herausforderungen und individuellen Ansprüchen, weiterzuentwickeln. Individualität wird zum gesellschaftlichen und pädagogischen Leitbegriff. (S. 589)

Dabei werden Biografien umso aufschlussreicher, je stärker sich ihre gesellschaftlich-lebensweltlichen Kontexte wandeln. Für die Phase des Alterns und für einen erziehungswissenschaftlichen Zugriff auf diese Phase bedeutet dies, dass das ,Lernen des Alterns selbst" im Verlauf der Zeit in den Mittelpunkt zu stellen ist. Dies folgt den Forderungen Sylvia Kades (2009), dass die notwendige fachliche Orientierung für die Zukunft der Altersbildung ${ }^{1}$ stärker als bisher auf kreative biografische Kompetenzen gelenkt werden muss. Allerdings nicht in einer didaktischen Auslegung dieses Ziels (z. B. im Sinne von Biografiearbeit mit älteren Menschen). Vielmehr sollten diese Selbsterzählungen für ein grundlagentheoretisches Projekt genutzt werden, das die erzählte individuelle Biografie als Ressource nutzt, um Biografien älterer Menschen zum Dreh- und Angelpunkt der Analyse von Bildungsprozessen älterer Menschen zu machen. Dies würde sich maßgeblich von der derzeitigen Debatte um Bildung und

\footnotetext{
${ }^{1}$ Zur Problematik der Benennung praktischer Bildungsarbeit mit älteren Menschen als Altenbildung, Geragogik, Bildung mit Älteren und Altersbildung vgl. Kade 2009, S. 51 ff.
} 
Alter unterscheiden: Entscheidend ist dort bislang die Differenzierung nach drittem und viertem Lebensalter. Dabei geraten die pädagogischen Modi Bildung und Hilfe in den Vordergrund mit der Leitdifferenz von Autonomie und Abhängigkeit (Kade 2009). Aber Lernbemühungen im Alter gestalten sich nicht automatisch altersspezifisch, sondern vielmehr biografie- und lebensphasenspezifisch. Diese Erkenntnisse sind sowohl aus der Gerontologie als auch der Erziehungswissenschaft (Nittel 2009) ableitbar. Dennoch ist in der Praxis der Altersbildung anderes geschehen: In einer Art Vorwärtsstrategie hat sie sich mit einem Zielgruppenprogramm vor allem an das ,autonome“ Alter gewandt (Kade 2009). ${ }^{2}$ Zudem fördert die praktische Altersbildung mit einer derartigen Entwicklung die Fantasie eines Steigerungsdiskurses, der die Problematiken des Schwundes und der Endlichkeit des Lebens nicht ernst nimmt (dazu auch Arnold 2006). Diesem Diktum folgend verfügen wir derzeit über dreierlei prominente „Akteure“ in der Debatte um Bildung im Alter: Didaktische Annäherungen, politische Aufforderungen und gerontologische Hintergrundtheorien, auf die auch im erziehungswissenschaftlichen Bezug auf Bildung immer wieder rekurriert wird (bspw. Bubolz-Lutz et al. 2010; Leipold 2012). Mit dem Lehrbuch Geragogik (Bubolz-Lutz et al. 2010) wird in der Definition von Bildung im Alter als Reflexion von Bildung und Handeln mit Subjekt-, Beziehungs- und Gesellschaftsorientierung vor allem ein didaktisches Programm aufgerufen, indem Bildung im Alter in unterschiedlichen Lernfeldern diskutiert wird und mögliche Methoden der Vermittlung angesprochen werden. Bedeutsam für die Altersbildung sind ebenso die Altenberichte der Bundesregierung. Insbesondere der 5. Altenbericht mit dem Titel „Potenziale des Alters in Wirtschaft und Gesellschaft“ (BMFSFJ 2006) hat großen Einfluss auf die Ausgestaltung von Bildungsprogrammen für das Alter hinsichtlich von Aktivierung und Verantwortungsübernahme. Die Altenberichte bestimmen maßgeblich die Debatte um das, was Bildung im Alter zu sein hat, mit, und zwar einerseits auf der programmatischen Ebene und andererseits auch auf finanzieller, fördernder Ebene, da die Berichte immer auch mit Projektförderungen verbunden sind (zu einer gesellschaftskritischen Auseinandersetzung mit diesem Thema Lessenich 2009; Denninger et al. 2014). Man könnte aber fragen, ob anhand dieser Papiere die Zielgruppe im Bildungsdiskurs richtig bestimmt oder nicht eher politisch erzeugt wird. So steht nach dem 5. Altenbericht beispielsweise die Frage im Raum, ob Ältere in derartigem Ausmaß bereit sind, sich bis ins hohe Alter ehrenamtlich zu engagieren. Eine weitere Referenz stellen die „klassischen“ gerontologischen Alternstheorien zum gelingenden Altern dar. Gelingendes Altern wird je nach Theorie sehr unterschiedlich ausgelegt. Durch Strategien, die das Individuum für gelingendes Altern benötigt, zeigen sich je nach Sichtweise in der Beibehaltung von Kontinuität (Atchley 1989), in der Anwendung von Selektion, Optimierung und Kompensation (Baltes 2007) und in der aktiven Gestaltung des Alters (Havighurst 1961) und auch im wechselseitigen gesellschaftlich-individuellen Rückzug aus sozialen Rollen (Cumming und Henry 1961) Strategien, die mit biografischen Strategien zum Lernen im Alter überlagert werden können. In diesen insbesondere entwicklungspsychologisch fundierten Theo-

\footnotetext{
${ }^{2}$ Auch wenn nun eine Hinwendung durch geragogische Angebote zum vierten Lebensalter erfolgt, wird dennoch der Autonomiegedanke nicht aufgegeben, und damit die Radikalität von Verlusten im Alter relativiert (Friese 2014).
} 
rien liegt aber auch verborgen, was erziehungswissenschaftlich in den Mittelpunkt gestellt werden sollte: Wie findet (gelingendes) Altern statt? Wie lernen wir in der Biografie Erworbenes für das nähere oder fernere Lebensende zu nutzen? Dennoch hinkt die Theoriebildung zum Themenbereich Altern und Bildung der praktischen Altersbildung hinterher. So wird bspw. in der Reaktion auf die Altenberichte etwas praktisch forciert, was theoretisch noch nicht geklärt ist, nämlich wie sich das Lernen des Alterns biografisch vollzieht und welche Bildungsprozesse dabei zum Tragen kommen. Zudem wissen wir noch zu wenig über die neuen Kohorten Älterer, die auf uns zukommen werden, über welche Bildungsziele sie verfügen und welche Zielgruppen hier praktisch wie theoretisch in den Blick genommen werden müssen. ${ }^{3}$ Bislang haben wir kaum eine Verknüpfung von erziehungswissenschaftlicher und gerontologischer Theoriebildung (Himmelsbach 2009a). So stehen beispielsweise die Begriffe „Bildung“ und „Entwicklung“ unverbunden nebeneinander, obwohl die oben angesprochenen, eher auf Entwicklung orientierten Strategien gelingenden Alterns auch als Aneignungsprozesse beschrieben werden könnten. Denn mit der Fokussierung auf das dritte Lebensalter setzt der bisherige Fokus von Alternsbildung vor allem auf Wachstum (Autonomie). Was das schwierige Unterfangen des Alterns ausmacht, was Baltes (2007) mit den drei Entwicklungszielen Wachstum, Aufrechterhaltung und Verlustregulation beschreibt, wird weitestgehend verschwiegen oder über den Modus Hilfe operationalisiert. Um sich dessen zu nähern, wie das „Altern selbst" gelernt wird, wäre eine Hinwendung zu den Aneignungsformen der Individuen selbst hilfreich: Ihre eigenen Narrationen des Älterwerdens verweisen auf ihre Bildungsgestalten.

\section{Anschluss an die Debatten der Bildungsbiografieforschung und deren Nutzen für eine erziehungswissenschaftlich orientierte Forschung zu Bildung im Alter}

Bildung wird sowohl als Prozess als auch als Gestalt angesehen und das Individuum stellt sich, um sich zu bilden, in ein Verhältnis zu sich selbst und zu seiner Umwelt (vgl. Langewand 2004). Die Auseinandersetzung von Ich und Welt als Bildung zu beschreiben, geht dabei zurück auf Humboldt ,so viel Welt als möglich zu ergreifen, und so eng, als er nur kann, mit sich zu verbinden“ (Humboldt 1969/1793, S. 235, zit. nach Kade 2012).

Bildung besteht mithin darin, dass die angeeignete Welt, insbesondere in der Form von Wissen über sie, ganz dem einzelnen Menschen zugehört. Durch diese weltoffene, nicht auf das kleine Selbst eines vereinzelten Menschen zurückgezogene Selbstbezüglichkeit wird das Ich einerseits zum autonomen Subjekt, andererseits entwickelt es sich nach der Seite seines Wissens, seiner Fähigkeiten und seines Könnens. (Kade 2012, S. 38)

Mit dieser dem Bildungsbegriff inhärenten Fokussierung auf das Individuum und seiner prozessuralen Gestaltbildung rückt zur Analyse von Bildungsprozessen die

${ }^{3}$ Soziologisch: van Dyk und Lessenich (2009), gerontologisch/soziale Arbeit: Aner et al. (2007). 
Biografie in den Mittelpunkt, um aus dieser heraus innewohnende Strukturen der Gestaltbildung zu analysieren. Mit der methodischen Figur der „Lebenskonstruktionen“ wird nach eben denjenigen Strukturen, die gestaltbildend für das Individuum sind, gesucht:

Von der Wiedererkennbarkeit einer „Gestalt“ im permanenten Wechsel und Wandel handelt der moderne Strukturbegriff. (...) Nicht innerer Zwang und mentale Vorschriften, sondern ein sich selbst konstituierendes System von Bedeutungen sichert die Identität der Person im Durchgang durch die verschiedenen Positionen im sozialen Raum und im Wechsel der biographischen Zustände. (Bude 1999, S. 248)

Dies gilt zunächst für die einmalige Biografieschilderung, die Zeitlichkeit im Verlauf der Schilderung des Lebens betrachtet. Die einzelnen Lebensereignisse und Lebenserfahrungen werden in einen inhaltlichen Zusammenhang gebracht, der das Werden der Person in den Mittelpunkt rückt (Alheit und Dausien 2007). Bildungsbiografische Prozesse erscheinen demnach als kontinuierliche, sinnhaft integrierte Prozesse der Veränderung und Transformation von Subjektzuständen und Subjektstrukturen durch die Aneignung von Welt. Sie sind damit aber auch verankert in einer gegenwärtigen Perspektive des Erzählens und können den dynamischen Prozess von Bildung im Hinblick auf Lebensalter, kontextueller Einbettung der gegenwärtigen Erzählung nur aus der erhobenen Gegenwart heraus beschreiben (Hof et al. 2012). Aber gerade eine solche performatorische Veränderung aus unterschiedlichen Erzählzeiten könnte für Fragen der Bildung im Alter von besonderem Interesse sein, denn bezogen auf das Individuum bedeutet Altern auch immer wieder das Verhandeln der Spannung zwischen der äußerlichen Erscheinung des Körpers und dem innerlichen subjektiven Prozess des ,wirklichen Selbst“, das paradoxerweise jung bleibt (Featherstone und Hepworth 1991). Gleichzeitig ist diese Spannung überlagert, wie beispielhaft am 5. Altenbericht erläutert, von gesellschaftlichen Zuschreibungsprozessen eines aktuell produktiven Alterns. Der Kontext, vor dessen Hintergrund Altern erzählt wird, könnte somit prägend für die Aneignungsprozesse des Alterns selbst sein.

Aktuell vollzieht sich eine Neuorientierung der Biografieforschung in Reaktion auf ein Zeit-, Welt- und Subjektdefizit. Kade und Hof (2010) konstatieren der Biografieforschung in den Suchbewegungen nach Gestaltbildung ein „Zeitdefizit“, da sich qualitative Biografie- und Bildungsbiografieforschung seit den 1980er Jahren der einmaligen Narration der Biografie widmet und damit unterschlägt, dass jede Bildungsbiografie ,nur eine performative Momentaufnahme in einem unendlichen Prozess des Biographisierens von Lebenslaufereignissen“ (Kade und Nolda 2014b) darstellt. Die Biografie kann es demnach gar nicht geben, sondern vielmehr eine „Serie bildungsbiographischer Gestalten im Lebenslauf“ (ebd.). Erst durch Analyse mehrerer biografischer Erzählungen und dem Vergleich dieser Erzählungen ist damit die Annäherung an die Gestaltbildung im Prozess der Bildung durch Rekonstruktion möglich. Andererseits steht das Verhältnis von Ich und Welt in der Kritik. In bisherigen Analysen wurde zu stark auf das Individuum und zu wenig auf die Welt, also die Kontexte, im Rahmen derer das Individiduum seine Bildungserfahrungen macht, fokussiert. So fordert Fuchs, nicht bei dem Biografieträger in den Analysen stehenzubleiben, sondern vielmehr, ,auch zu berücksichtigen, was die sozialen Verhältnisse 
bedeuten, auf welche Weise sie wirken und wie sie in die Biographie verwoben sind“" (Fuchs 2012, S. 173 f.). Hinsichtlich eines Subjektdefizits betont Koller die klassische Hinwendung zu „Kräften“ und bemängelt die Ausblendung von Konflikten, Widersprüchen, Verlusterfahrungen und Stillstand beim ,,anders werden mit offenem Ausgang“ (Koller 2012, S. 169).

Durch diese Neuorientierung ergeben sich Chancen für einen neuen Zugriff auf das Phänomen „Bildung im Alter“: Altern im Vollzug beobachtbar zu machen und als Bildungsprozess zu beschreiben. Doch für diesen Zugang bedarf es eines Datensatzes, der das Beobachten des „Altern Lernens“ über die Zeit ermöglicht. Mit der ILSE-Studie liegt ein solcher Datensatz vor.

\section{Rückgriff - Bildungsbiografische Sekundäranalyse - Die Interdisziplinäre Längsschnittstudie des Erwachsenenalters (ILSE)}

Die zu Beginn der 1990er Jahre initiierte „Interdisziplinäre Längsschnittstudie des Erwachsenenalters" (ILSE) formuliert als Ziel die Untersuchung individueller, gesellschaftlicher und materieller Bedingungen für ein gesundes, selbstverantwortliches und zufriedenes Altern. Bislang sind drei Erhebungswellen abgeschlossen und eine vierte Welle befindet sich derzeit in Erhebung. ${ }^{4}$ Als interdisziplinäre Längsschnittstudie (Disziplinen: Entwicklungspsychologie, Körpermedizin, ökologische Gerontologie, Gerontopsychiatrie, Psychosomatik, Zahnmedizin, Medienforschung) bietet die ILSE-Studie neben der Analyse inter- und intraindividueller Unterschiede und Veränderungen über die Lebensspanne auch die Möglichkeit, Zusammenhänge zwischen Umweltfaktoren, Verhaltensaspekten, Lebensereignissen, Gesundheitsverhalten, der psychischen und physischen Gesundheit sowie dem Wohlbefinden zu untersuchen. Der in der ILSE-Studie verfolgte biografische Ansatz („Biographische Exploration“) basiert dabei auf der Annahme, dass die Qualität von Erfahrungen in frühen Lebensphasen die Adaptationsleistungen und -möglichkeiten in späteren Lebensphasen in einzigartiger Weise mitbestimmen (Martin et al. 2000). Die ILSEStichprobe bestand zum ersten Messzeitpunkt aus 1.390 Personen aus Ost- und Westdeutschland. Diese wurden nach den Stratifizierungsdimensionen Geschlecht und Kohortenzugehörigkeit zu den Jahrgängen 1930-1932 (K30) bzw. 1950-1952 (K50) repräsentativ ausgewählt. Der erste Untersuchungsdurchgang wurde 1993-1996, der zweite Messzeitpunkt ( $N=898$ Personen) wurde 1997-2000 durchgeführt. Der seit Januar 2005 laufende dritte Untersuchungsdurchgang wurde 2007 abgeschlossen $(N=670)$ (BMFSFJ 2010). Eine vierte Untersuchungswelle läuft aktuell (Tab. 1).

\footnotetext{
${ }^{4}$ Die Daten dieser Arbeit basieren auf der Interdisziplinären Längsschnittstudie des Erwachsenenalters (ILSE). Für die Möglichkeit, diese Daten für meine Forschungsfragestellung auszuwerten, bedanke ich mich an dieser Stelle sehr herzlich. ILSE wurde in Bezug auf ihre ersten drei Messzeitpunkte aus Mitteln des Bundesministeriums für Familie, Senioren, Frauen und Jugend und des Landes Baden-Württemberg finanziert. Die von Prof. Dr. Ursula Lehr und Prof. Dr. Hans Thomae gestartete Studie wurde in den Jahren 1993 bis 2000 am Deutschen Zentrum für Alternsforschung an der Universität Heidelberg, Abt. Entwicklungsforschung, organisiert und koordiniert. Der dritte Messzeitpunkt (2005-2007) wurde am Psychologischen Institut, Abteilung für Psychologische Alternsforschung, durchgeführt. Der vierte Messzeitpunkt (2014-2015) erfolgt derzeit an der Universität Heidelberg unter der Leitung von Dr. Christine Sattler, Prof. Dr. Johannes Schröder und Prof. Dr. Hans-Werner Wahl mit Förderung durch die Dietmar Hopp Stiftung.
} 
Tab. 1 Stichprobenbeschreibung ILSE Studie über die ersten drei Messzeitpunkte (vgl. BMFSFJ 2010, S. 21)

\begin{tabular}{|c|c|c|c|c|c|c|}
\hline \multirow{2}{*}{$\frac{\text { Messzeitpunkt (1) }}{\text { Zeit seit T1 }}$} & \multicolumn{2}{|c|}{ T1 (1993-1996) } & \multicolumn{2}{|c|}{ T2 (1997-2000) } & \multicolumn{2}{|c|}{ T3 (2005-2007) } \\
\hline & & & ca. 4 Jahre & & ca. 12 Jahre & \\
\hline Zeit seit T2 & & & & & ca. 8 Jahre & \\
\hline Geburtskohorte & 1930/1932 & $1950 / 1952$ & $1930 / 1932$ & $1950 / 1952$ & 1930/1932 & $1950 / 1952$ \\
\hline Region West & 393 & 391 & 223 & 224 & 150 & 168 \\
\hline Region Ost & 302 & 304 & 226 & 225 & 168 & 184 \\
\hline N Kohorte (je MP) & 695 & 695 & 449 & 449 & 318 & 352 \\
\hline N gesamt (je MP) & 1390 & & 898 & & 670 & \\
\hline
\end{tabular}

Um über eine Nutzung der Daten aus sekundäranalytischer qualitativer Sicht eingehen und auch die Chancen und Grenzen des Materials beschreiben zu können, bedarf es noch der Explikation eines zentralen Erhebungsformats der ILSE-Studie: der Biographischen Exploration. Von allen Interviewteilnehmern über alle Messzeitpunkte hinweg wurden Tonbandaufzeichnungen des Teils der Erhebung angefertigt, der sich mit der Biographischen Exploration beschäftigt. Diese kommt nur zu T1 zum vollen Einsatz und erzeugt Aufzeichnungsmaterial eines Individualinterviews von bis zu sechs Stunden. In den beiden darauffolgenden Erhebungen wird der Teil der Biographischen Exploration zunehmend standardisiert und der offene Teil der Befragung bezieht sich vor allem auf Veränderungen seit dem letzten Interview. Diese Veränderungen werden halboffen zu den Themen Veränderungen allgemein, Gesundheit, finanzielle Situation, soziales Netzwerk, Zukunft und Lebensrückblick erfragt. Diese Aufzeichnungen sollen nun erstmals einer qualitativen, am Text orientierten, rekonstruktiv angelegten Analyse zugänglich gemacht werden. ${ }^{5}$

\subsection{Spezifizierung: Die Methode der Biographischen Exploration}

Ziel der Methode der Biographischen Exploration ist es, das „Individuum in seiner Welt“ zu erfassen (Thomae 1987, 1996). Das Verfahren dient dem Zweck, Konstanz und Veränderung im Erleben und Verhalten in bedeutsamen Entwicklungsabschnitten des Individuums zu beschreiben (Kruse 1987). Vom methodologischen Anspruch vereint dieses Verfahren idiografische und nomothetische Forschungsprinzipien. Dabei liegt der idiografische Ansatz insbesondere im Erhebungsverfahren begründet, und der nomothetische in der Auswertungsstrategie. Thomae selbst ordnet das von ihm entwickelte Erhebungsverfahren als halb-strukturiertes Interview ein. So soll das biografische Material des Individuums möglichst vollständig, natürlich und begrifflich adäquat abgebildet werden. Thomae geht allerdings nicht von der Eigenkonstruktivität im Moment des Erzählens der Biografie aus, sondern von einer ,wahren“ Biografiedarstellung. In seinen Ausführungen zur Biographischen Exploration schließt er gar die Auswertung von Biografien aus, die zu starke Verzerrungen oder Verdrehungen der Fakten darböten (Thomae 1987, S. 114). Damit bleibt Thomae von der methodologischen Fundierung her ,nomothetisch“ begründet, was auch erklären mag, dass beispielsweise die Leitfäden im Projekt ILSE strikt chronologisch angelegt

\footnotetext{
${ }^{5}$ Erziehungswissenschaftlich, aber weitestgehend auf Basis der quantitativen Daten, wurde die Studie von Iller (2006) genutzt, um berufliche Weiterbildung der K30 zu analysieren.
} 
sind. Noch strikter nomothetisch argumentiert Thomae bezüglich der Auswertung der Daten. Ein überindividuelles, auf Quantifizierung hin gedachtes Auswertungsmodell, wird verfolgt mit dem Versuch, Verbindungen zwischen den individuellen Welten herzustellen, um allgemeingültige Aussagen zu formulieren. Aus diesem Grund lehnt er die Interpretation (,,impressionistische Auswertung“, Thomae 1987) des biografischen Materials ab und setzt vielmehr auf eine Art komplexer Kategorisierung (im Sinne der von ihm entwickelten Reaktionsformen).

\subsection{Chancen und Grenzen des qualitativen Materials der ILSE-Studie}

Mit diesen methodischen Grundlagen ausgestattet unterliegt das enorm umfangreiche Material der ILSE-Studie Chancen und Grenzen hinsichtlich einer rekonstruktiv angelegten und erziehungswissenschaftlich orientierten Auswertung der Biografien. Als größte Chance ist die vorhandene Mehrfacherhebung über einen Zeitraum von über (bezieht man die aktuell laufende T4-Erhebung mit ein) 20 Jahren mit vier Erhebungswellen und jeweiliger Aufzeichnung der Daten zu bewerten. Für diesen Zeitraum ist auch die Auswahl der Kohorten bedeutungsvoll, lässt sich doch an der K30 über den Verlauf der 20 Jahre der Übergang vom dritten ins vierte Lebensalter nachvollziehen. Stellt man den Begriff der Generation (Schäffer 2012) ins Zentrum, ist es bedeutungsvoll, dass nun zum Zeitpunkt T4 die K50 das Alter von K30 zu T1 erreicht haben und sich damit auch am Übergang ins dritte Lebensalter befinden. Damit bieten sich vielfältige Möglichkeiten für Zugänge zu Individualitätsperformanz über die Zeit. Aber auch hinsichtlich der Kontextkonstellationen (Kade und Nolda 2014a) bieten sich in diesem Datensatz vielfältige Möglichkeiten; eine Besonderheit stellt dabei die Ost-West-Stratifizierung des Datensatzes dar. Die Reichhaltigkeit der Kontextualisierungsmöglichkeiten soll Abb. 1 veranschaulichen, sind doch die beiden

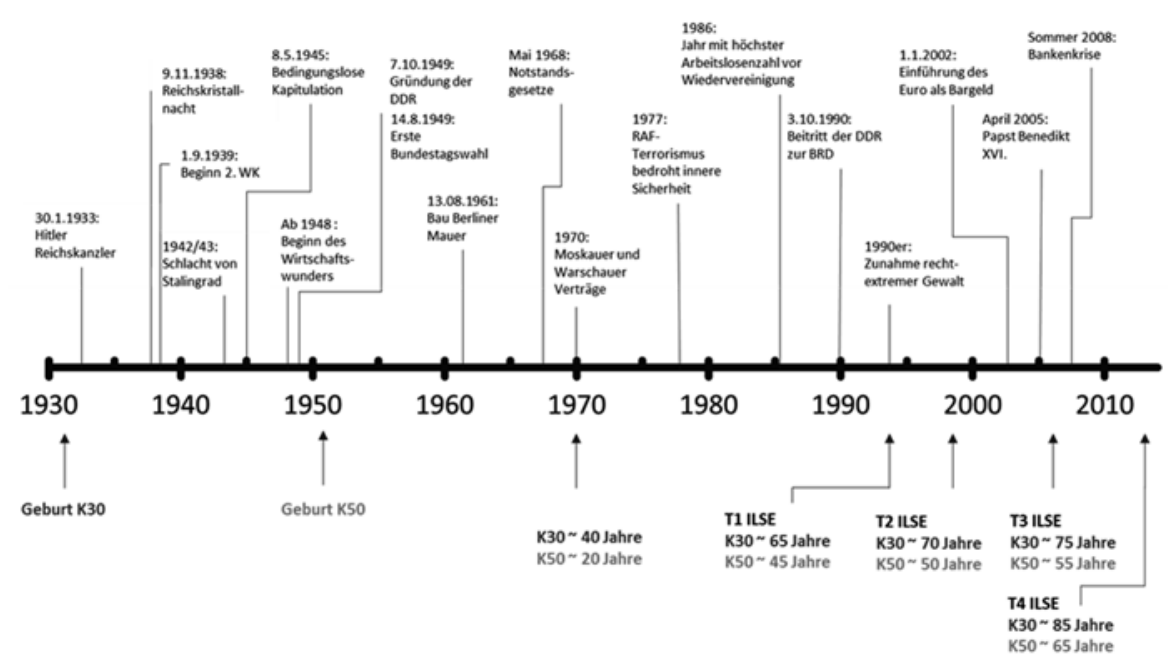

Abb. 1 Übersicht über die Erhebungszeiträume der ILSE-Studie in Verbindung mit ausgewählten Daten der deutschen Geschichte der vergangenen 85 Jahre 
Kohorten in ganz unterschiedlicher Weise (Lebensalter, Orte, Voraussetzungen) in die historisch-gesellschaftlichen Zusammenhänge verwoben.

Das Material ist nicht nur chancenreich, sondern auch herausfordernd. So sind die Daten nicht unter einer bildungstheoretischen Fragestellung erhoben worden. In Anlehnung an die von Kade und Nolda (2014a) vorgeschlagene Mehrfacherhebung muss als Grenze des Materials auch die Einschränkung gelten, dass keine vollständigen Biografieerhebungen zu allen Messzeitpunkten vorliegen. Da der ursprüngliche Auswertungsfokus eben gerade nicht auf der Rekonstruktion von Biografien lag, wurde die Erhebung zunehmend standardisiert und es liegen zu den späteren Messzeitpunkten nur noch Fragen zu Veränderungen seit dem letzten Interview vor (das unten dargestellte Fallbeispiel stellt einen Versuch des Umgangs mit dieser Realität dar). Als weitere Herausforderung stellt sich der für ein qualitatives Vorhaben immense Umfang des Datensatzes dar.

\subsection{Möglicher Zugriff auf das Material mit erziehungswissenschaftlicher Fokussierung}

Die Auswahl der Fälle wird in hohem Maße selektiv sein. Zudem soll nur aus dem Material geschöpft werden, zu dem alle Zeitpunkte vorliegen und keine kognitiven Beeinträchtigungen der Personen eingetreten sind. In einer ersten Runde der Auswertungen sollen Fallanalysen unter spezifischen Individualitäts- und Kontextvariationen im Fokus sein, beispielsweise der Vergleich von Frauen der K30 in Ost und West oder der Vergleich von K30 und K50. Eine erste Analyse erfolgt unter Auswahl eines spezifischen Kontextes, den weiter unten beschriebenen Narrativen des Alterns (Lessenich 2014) (siehe Fallbeispiel).

Im Zugriff auf das Material sollen von den Interviewten geschilderte Veränderungen zur analytischen Schaltzentrale gemacht werden, was gegenstandsangemessen im Hinblick auf die in ILSE vollzogene Erhebungsstrategie erscheint. Veränderungen wurden in allen Erhebungen erfragt und können als zentral für das Aufdecken von Bildungsprozessen erachtet werden. Zudem ist der Prozess abhängig von der historischen Situation und dem Stand pädagogischer Formenbildung zu einem erfragten Zeitpunkt (bspw. T1). Dieser Bildungszustand verändert sich zu jedem erhobenen Zeitpunkt (bspw. T3). Veränderte Bildungszustände sind aber auch Folgen von signifikanten Lebensereignissen, Übergängen in der Biografie oder Krisenerfahrung. Bildung stellt demnach einen kontinuierlich-diskontinuierlich verlaufenden Prozess dar (Kade 2012, S. 43). Hinzu kommt in Anlehnung an Kollers Vorschlag einer Theorie transformativer Bildungsprozesse, dass Bildung nicht nur als Kompetenzerwerb, sondern vielmehr als eine grundlegende Veränderung der gesamten Person zu begreifen ist, der auch Scheitern und Verlustregulation immanent sein können. Mithilfe von Bildung wird auf neue Problemlagen reagiert, die mit den verfügbaren Mitteln nicht mehr angemessen bearbeitet werden können (Koller 2012).

Erste Suchbewegungen im Material gehen aus diesen Gründen von dem Interviewzeitpunkt T3 aus, und versuchen, die dort geschilderten Veränderungen zunächst im T3-Interview und anschließend in Relation zum T1-Interview zu erklären. 


\section{Altern lernen im Narrativ des Ruhestands - Ein Fallbeispiel zur biografischen Kontinuität von Pflege- und Sorgebeziehungen}

Das Fallbeispiel verwebt individuell geschilderte Veränderungen der Person mit einem exemplarischen Kontext: den drei von Lessenich (2014) beschriebenen Narrativen des Alterns. Als Ergebnis einer Diskursanalyse erschließt er diese aus sozialpolitischen Debatten um erhöhten Leistungs- und Verantwortungsanforderungen an ältere Menschen. Dies sieht er in engem Zusammenhang mit der Rezeption insbesondere kompetenzorientierter gerontologischer Alternstheorien (Denninger et al. 2014; Lessenich 2014): Das Narrativ des Ruhestands (als Narrativ der 1980er Jahre) erscheint als negativer Gegenhorizont neuer Altersbilder: In der Vorstellung vom ruheständigen Alter verschränken sich das Wissen um die Erwerbsentpflichtung mit materieller Versorgung des Alters über die Institution der gesetzlichen Rentenversicherung mit Assoziationen des Rückzugs aus dem gesellschaftlichen Leben und der sozialen Vereinzelung, des körperlichen Abbaus und des Nachlassens von außerhäuslichen Aktivitäten, zuweilen als sinnentleert und wenig freudvoll. Das Narrativ des „Unruhestands“ (Narrativ der 1990er Jahre) greift die Erkenntnisse zur Plastizität des alternden Körpers und des alternden Hirns auf und legt den Fokus auf die Kompetenzen und die Gestaltbarkeit des Alters im Sinne einer, ,individuell erfolgreichen Langlebigkeit“. Es verschafft dem Individuum die Möglichkeit, sich als ,junger“ alter Mensch zu konstituieren. Als drittes sieht Lessenich mit dem 5. Altenbericht das Narrativ des produktiven Alters gekommen, das Ältere mehr und mehr in die Verantwortung nimmt. Es schreibt das Narrativ des Unruhestands fort und um, indem nicht länger nur der Selbstzweck des erfolgreichen Alterns betont wird, sondern die Aufforderung hinzukommt, etwas für andere zu tun und/oder durch eigene Tätigkeit (Bsp. „Rente mit 67“) dem Strukturwandel des Alters (Tews) zu begegnen. Ältere werden nun als nützliche, wertschaffende und ertragreiche Ältere konzeptualisiert (Lessenich 2014, S. 456 f.). ${ }^{6}$

In einem ersten Zugriff auf das Material wird nun der Frage nachgegangen, ob die dargelegten Veränderungen zum Zeitpunkt T3 in Relation zu den Schilderungen zu T1 stehen und wie sie mit den drei Narrativen in Verbindung zu bringen sind.

Frau M. ist 1932 geboren und entstammt einem ländlich-bäuerlichen Milieu. Frau M. hat einen Volksschulabschluss und keine Berufsausbildung. Ihr Wunsch war, Schneiderin $\mathrm{zu}$ werden, und eine fortgesetzte Schulbildung wird immer wieder als Sehnsuchtsmotiv kenntlich gemacht, aber nie realisiert. Frau M. heiratet ihren drei Jahre jüngeren Ehemann im Jahr 1957. Sie haben drei gemeinsame Kinder. Der erste uneheliche Sohn kommt erst nach zehn Ehejahren in die bereits mit dem Ehemann gegründete Familie. Die Ehe wird als ambivalent geschildert, da ihr Mann trinkt. Sie lebt zu beiden Interviewzeitpunkten gemeinsam mit ihrem Ehemann in einer Mietswohnung in einer größeren Stadt. Frau M. arbeitet ihr Leben lang und kennzeichnet

\footnotetext{
${ }^{6}$ In seinen 2009 und 2014 dargelegten Analysen geht Lessenich dabei noch wenig darauf ein, wie dieser Diskurs bei den Älteren selbst ankommt, sprich in deren Selbstbeschreibungen auftaucht. Dies realisiert hingegen die Analyse von Denninger et al. (2014) in der Anwendung auf das ,junge Alter“, das dritte Lebensalter (Interviewpartner aus den Geburtsjahrgängen 1938-1952). Das vierte Alter wird von Lessenich zunächst als im Diskurs wegerklärtes Phänomen dargelegt, aber auch noch in der empirischen Analyse von Denninger et al. (2014) weitestgehend ausgespart.
} 
ihre Biografie als Arbeitsbiografie, selbst in Passagen, die im Interview als Phasen der Kindererziehung und Hausfrauentätigkeit gekennzeichnet werden, hat sie nebenher mehrere Putzstellen, um das Familieneinkommen aufzubessern und Phasen der Erkrankung des Ehemannes zu überbrücken. Das erste Interview im Rahmen einer Biographischen Exploration (T1) fand im Jahr 1994 statt, als Frau M. 62 Jahre alt war. Berufsbiografisch stand sie etwa ein Jahr vor dem Ruhestand, während ihr Mann schon in den Ruhestand versetzt war. Das zweite Interview, das in den Blick genommen wird (T3), wurde im Jahr 2005 durchgeführt, also im Alter von 73 Jahren. Frau M. ist zu diesem Zeitpunkt im Ruhestand und pflegt ihren schwer pflegebedürftigen Mann.

Die zugrunde liegende Blickrichtung dieses Porträts wird bestimmt von den Veränderungen von T3 zu T1. Die allgemeine Frage nach Veränderungen stellt gleichzeitig den Einstieg in das Interview zum Zeitpunkt T3 dar. Die erste und wesentliche Veränderung von 1994 zu 2005 ist die Pflegebedürftigkeit des Ehemanns, mit der Frau M. in das Interview einsteigt. Sie überlagert jeden mithilfe des Leitfadens angesprochenen Themenbereich (Gesundheit, finanzielle Situation, Wohnen, soziales Netzwerk, Zukunft). Alle Fragen, die vom Interviewer eigentlich in Richtung der Veränderung und Ansichten von Frau M. adressiert sind, münden in unterschiedlichen Perspektivnahmen auf die Pflegebedürftigkeit des Ehemannes, so bspw. im Themenbereich Gesundheit: Die eigenen zunehmenden körperlichen Beeinträchtigungen werden nur randläufig erwähnt; Prävention, selbst in Form von Spaziergängen, wird nicht realisiert, da sie mit dem Mann ,ihre Arbeit“ habe. Es entsteht der Eindruck eines ,passiven“ vierten Lebensalters. Sie selbst hat noch wenige Beeinträchtigungen, aber durch ihre enge und (fast) auf sich allein gestellte Pflege des Ehemanns präsentiert sie ein Leben ohne große Abwechslung, geprägt von sozialer Zurückgezogenheit (für das Treffen mit Freundinnen ist keine Zeit) und Sorge um die Zukunft. Die Ehe wird als schwieriger erlebt, mit einem Pflegefall sei es, als ob man ein Kind zu erziehen habe, aber „Krankheit bindet eben mehr“, sowohl hinsichtlich der eingesetzten Ressourcen als auch emotional.

Setzt man dieses ,passive“ vierte Lebensalter nun in Beziehung zu T1, stellt sich heraus, dass Sorgen und Pflegen seit Kindestagen zentrale Lebensthemen darstellen. Die fast lebenslange Abkehr von formalen Bildungsprozessen taucht nur in der rückblickenden Sehnsucht nach einem höheren Bildungsabschluss auf, dafür aber mehrfach und in beiden Interviewsettings. Aber in der Biografie Frau Ms. bleibt dafür nie Zeit; zu drängend sind die Problemlagen der elterlichen und später der eigenen Familie. Wenn Weiterbildungen besucht werden, dann nicht für einen beruflichen "Verwendungszweck“, sondern zur Unterstützung der Kinder in der Schule oder zur Aufbesserung der familiären finanziellen Situation (Schreibmaschinenkurs); letztlich sind also die wenigen formalen Bildungsaktivitäten auch als Sorgebeziehungen zu verstehen.

Dass und wie sich Sorgebeziehungen entwickeln, verdeutlicht ein Interviewausschnitt aus T3, der unter Verwendung der gleichen Motive (Krankheit der Mutter, Geburt der jüngsten Schwester, englische Krankheit und eigenständiges Entscheiden gegen eine Lehre) im T1-Interview auftaucht. Zudem ist es der einzige explizite und ausführliche biografische Rückgriff im T3-Interview: 
I: Sie haben schon gesagt, also Ihre Tochter unterstützt Sie manchmal bei der Pflege vom \# falls sie mal [*] rüberkommt für so eine Stunde. P: [*] Ja ab und zu ja. I: ist es auch so dass sie Sie mal tröstet, wenn es Ihnen mal nicht so gut geht, also gibt es auch [*] so... P: [*] Ja körperlich [äh] ich habe können keine Liebe spenden, ich habe meine Kinder auch \# ich bin so erzogen worden (--) und die von der Erziehung (--) kann man das praktisch nicht weiter \# wenn sie mal komm her Kind oder sonst ich war die Älteste (---) und das war eine schwere Zeit, weil meine Mutter war immer krank (--) und 47 bin ich aus der Schule gekommen und [äh] (---) 47 bin ich aus der Schule gekommen ja (2.0) und da war ein Jahr noch 4849 bin ich erst zum Arbeiten gegangen (--) da habe ich müssen meine Mutter unterstützen [...] und [äh] mit vier Kinder die kleinste auf die Welt gekommen da unten (---) und da hat sie Ansatz gehabt von Englischkrankheit (---) und da hat man ja als fünfzehn Jahre älter hat man ja müssen Mutter unterstützen (--) in allem war ich [äh] fünfzehn Jahre äl/\# war ich gerade fünfzehn bin ich aus der Schule gekommen und da hat meine Mutter gesagt du gehst jetzt in die Le/also in die Lehre zum Nähen oder was, aber die hat keine keine Prüfung gehabt (--) und da hat sie gesagt du gehst trotzdem hin wenn du später deinen Kindern was nähen willst. (Frau M., T3)

Die Rolle der ältesten Schwester und die Notwendigkeit der Unterstützung der Mutter nach der Geburt des jüngsten Kindes scheint hinsichtlich der als Arbeit definierten Sorgebeziehungen eine zentrale Schlüsselerzählung Frau M.s zu sein. Obwohl die Mutter die Lehre in Aussicht stellt, fühlt sie sich verpflichtet, zu sorgen. Der Abbruch der formalen Bildungsbemühungen stellt sich als unverrückbare familiäre Notwendigkeit dar. Diese Erzählung stellt gleichzeitig den Beginn der durch Sorge und Pflege geprägten Biografie wie auch den zentralen Schaltpunkt hinsichtlich der Bildungsbiografie dar. Pflege und Fürsorge setzen sich in der Biografieerzählung zu T1 kontinuierlich fort, mit Bezug auf die Kinder, die Enkel, der Pflege der Schwiegereltern und schließlich des eigenen Ehemannes, immer changierend zwischen Notwendigkeiten des Pflegens und der finanziellen Sorge. Gleichzeitig wird dieses Sorgen nicht nur fürsorglich ausgelegt, wie die Aussage ,ich habe keine Liebe spenden können" eindrücklich darlegt. Sorgen und Pflegen mit all seinen Ambivalenzen wird als ihre Lebensarbeit bezeichnet. Im Hinblick auf Altern lernen bedeutet das lebenslange Sorgen zunächst Fortsetzung, aber auch Negierung des eigenen zunehmenden Alters. Sie ist einerseits in eine passive Rolle gedrängt durch die zeitaufreibende und anstrengende Pflege des Ehemannes, vermeidet damit aber andererseits, sich ihrem eigenen Alternsprozess zu stellen.

Frau Ms. Schilderungen passen zunächst ins Ruhestands-Narrativ. Altern wird zurückgezogen und selbstbezogen, auch mit Vereinzelungstendenzen (T3) gelebt mit der baldigen (T1) Erwerbsentpflichtung mit materieller Versorgung durch die gesetzliche Rentenversicherung. Allerdings im Jahr 2005 und nicht als negativer Gegenhorizont zu neueren Altersbildern, sondern als aktuelle Realität. Unruhestand, wie im Narrativ von Lessenich formuliert, findet nicht in seiner Konnotation statt. In dieser taucht er nur einmal schlaglichtartig im Wunsch nach Reisen im Ruhestand (T1) auf. Unruhestand herrscht aber dennoch vor, aber eher konnotiert mit der permanenten Fortsetzung der Lebensarbeit und jenseits des Bildes des Rufs nach Aktivität und immer wieder erneuter Lebenskraft. Obwohl dieses Narrativ im Jahr 2005 bereits 
„wirkmächtig“ sein sollte, spielt es im Sinne einer Neuerfindung eines jungen Alters im Leben von Frau M. keine Rolle. Auch das Narrativ des produktiven Alters wird im Falle von Frau M. verkehrt, so ist sie doch eine „nützliche Alte“ (Lessenich); schließlich übernimmt sie durch die häusliche Pflege gesellschaftliche Verantwortung, aber daneben engagiert sie sich nicht ehrenamtlich und entlastet nicht durch fortgesetzte Erwerbstätigkeit die Alterssicherungssysteme, vielmehr setzt sie die eigene biografisch erworbene Sorgestrategie im Alter weiter fort.

Ein erster Einblick in das Material kann also dahingehend beurteilt werden, dass in diesem Fall die Narrative eher Proklamationen an die Gesellschaft darstellen, und dass sich Altern hier vielmehr als janusköpfige Realität zeigt, die eben das zunehmend abhängige kranke Altern nicht zu verstecken vermag, wenn es auch noch nicht ganz bei Frau M. selbst angekommen ist.

\section{Konsequenz - Das weitere Vorgehen, Fragen der biografischen Bildungsforschung im Alter}

Bei diesem Fallbeispiel und den dargelegten Ableitungen hinsichtlich der Formulierung einer Fragestellung handelt es sich um erste Annäherungen, die weiterer Präzisierungen bedürfen. Nächste Schritte sind dabei eingehendere Analysen an kontrastierenden Fällen zur Frage der Narration und Kontexte um Bildungsbiografien mit sozialpolitisch evozierten Narrativen des Alterns. Zudem bedarf es der Hinzuziehung weiterer Kontexte, wie beispielsweise dem Bezug auf historisch-gesellschaftliche Kontexte, um die Spezifität des „Altern Lernens“ zu ergründen.

Sofern schon auf Ergebnisse hingedacht werden kann, könnten diese einen Beitrag zu einer amalgierenden Theoriebildung von Gerontologie und Erziehungswissenschaft beitragen, womit, so die Hoffnung, letztendlich auch zukünftige Zielgruppen von Bildung im Alter besser beschrieben werden könnten und dies ohne das Differenzial Bildung und Hilfe im Sinne einer pädagogischen Übersetzung von drittem und viertem Lebensalter anzuwenden, sondern unter der empirisch fundierten Annahme über das, was das „Altern Lernen“ in seiner Komplexität ausmacht.

Open Access Dieser Artikel unterliegt den Bedingungen der Creative Commons Attribution License. Dadurch sind die Nutzung, Verteilung und Reproduktion erlaubt, sofern der/die Originalautor/en und die Quelle angegeben sind.

\section{Literatur}

Alheit, P., \& Dausien, B. (2002). Bildungsprozesse über die Lebensspanne und lebenslanges Lernen. In R. Tippelt (Hrsg.), Handbuch Bildungsforschung (S. 565-585). Opladen: Leske + Budrich.

Alheit, P., \& Dausien, B. (2007). Lifelong learning and biography: A competitive dynamic between the macro- and the micro level of education. In W. Linden, P. Alheit, A. S. Andersen, \& B. Merill (Hrsg.), Using biographical and life history approaches in the study of adult and lifelong learning: European perspectives (S. 57-70). Frankfurt a. M.: Verlag Peter Lang.

Aner, K., Karl, F., \& Rosenmayr, L. (Hrsg.). (2007). Die neuen Alten. Retter des Sozialen? Wiesbaden: VS Verlag. 
Arnold, R. (2006). Abschiedliche Bildung: Anmerkungen zum erwachsenenpädagogischen Verschweigen des Todes. Report. Zeitschrift für Weiterbildungsforschung, 3, 19-28.

Atchley, R. C. (1989). A continuity theory of normal aging. The Gerontologist, 2, 183-190.

Baltes, P. B. (2007). Alter(n) als Balanceakt: Im Schnittpunkt von Fortschritt und Würde. In P. Gruss (Hrsg.), Die Zukunft des Alterns (S. 15-34). München: Beck.

Bubolz-Lutz, E., Gösken, E., Kricheldorff, C., \& Schramek, R. (2010). Geragogik: Bildung und Lernen im Prozess des Alterns. Stuttgart: Kohlhammer.

Bude, H. (1999). Lebenskonstruktionen als Gegenstand der Biographieforschung. In G. Jüttemann \& H. Thomae (Hrsg.), Biographische Methoden in den Humanwissenschaften (S. 247-258). Weinheim: Beltz.

Bundesministerium für Familie, Senioren, Frauen und Jugend (BMFSFJ). (2006). Potenziale des Alters in Wirtschaft und Gesellschaft. Der Beitrag älterer Menschen zum Zusammenhalt der Generationen: Fünfter Bericht zur Lage der älteren Generation in der Bundesrepublik Deutschland. http:// www.bmfsfj.de/RedaktionBMFSFJ/Abteilung3/Pdf-Anlagen/fuenfter-altenbericht,property=pdf, bereich=bmfsfj,sprache=de,rwb=true.pdf. Zugegriffen: 07. Nov. 2014.

Bundesministerium für Familie, Senioren, Frauen und Jugend (BMFSFJ) (Hrsg.). (2008). Interdisziplinäre Längsschnittstudie des Erwachsenalters. Abschlussbericht anlässlich der Fertigstellung des dritten Messzeitpunktes. Berlin: freizugängliches pdf.

Cumming, E., \& Henry, W. E. (1961). Growing old: The process of disengagement. New York: Basic Books.

Dausien, B. (2014). „Bildungsentscheidungen“ im Kontext biografischer Erfahrungen und Erwartungen. Theoretische und empirische Argumente. In I. Miethe, J. Ecarius, \& A. Tervooren (Hrsg.), Bildungsentscheidungen im Lebenslauf: Perspektiven qualitativer Forschung (S. 39-61). Opladen: Verlag Barbara Budrich.

Denninger, T., van Dyk, S., Lessenich, S., \& Richter, A. (2014). Leben im Ruhestand: Zur Neuverhandlung des Alters in der Aktivgesellschaft. Bielefeld: Transcript.

Featherstone, M., \& Hepworth, M. (1991). The mask of aging and the postmodern life-course. In M. Featherstone, M. Hepworth, \& B. S. Turner (Hrsg.), The body. Social process and cultural theory (S. 371-389). London: Sage.

Friebe, J., Schmidt-Hertha, B., \& Tippelt, R. (2014). Kompetenzen im höheren Lebensalter: Ergebnisse der CILL-Studie. Bielefeld: W. Bertelsmann Verlag.

Friese, A. (2014). Bildungsressourcen hochaltriger Menschen im Alten- und Pflegeheim. Eine qualitative Studie zu vorhandenen Ressourcen und faktischer Bildungsbeteiligung von Menschen im hohen Lebensalter durchgeführt in einer stationären Senioreneinrichtung (S. 38-56470). Köln urn:nbn:de:hbz: Dissertation.

Fuchs, T. (2012). Bildungstheorie und Bildungsforschung - Die Etablierung von „Annäherungsarenen“ im Kontext qualitativer Forschung. In I. Miethe \& H. R. Müller (Hrsg.), Qualitative Bildungsforschung und Bildungstheorie (S. 129-147). Opladen: Verlag Barbara Budrich.

Havighurst, R. J. (1961). Successful aging. The Gerontologist, 1, 8-13.

Himmelsbach, I. (2009a). Altern zwischen Kompetenz und Defizit. Wiesbaden: VS Verlag.

Himmelsbach, I. (2009b). Bildung im Alter in sozialen Welten: Diesseits und jenseits von Dichotomien. Zeitschrift für Erziehungswissenschaft, 3, 457-473.

Hof, C., Kade, J., \& Fischer, M. E. (2012). Serielle Bildungsbiographien. Auf dem Weg zu einem qualitativen Bildungspanel zum Lebenslangen Lernen. Zeitschrift für Pädagogik, 3, 328-339.

Iller, C. (2006). Altern gestalten - berufliche Entwicklungsprozesse und Weiterbildung im Lebenslauf. http://www.die-bonn.de/esprid/dokumente/doc-2005/iller05_12.pdf. Zugegriffen: 19. Nov. 2014.

Kade, S. (2009). Altern und Bildung: Eine Einführung. Bielefeld: W. Bertelsmann.

Kade, J. (2012). Bildungstheorie und Bildungsforschung. In B. Schäffer \& O. Dörner (Hrsg.), Handbuch Qualitative Erwachsenen- und Weiterbildungsforschung (S. 37-49). Opladen: Verlag Barbara Budrich.

Kade, J., \& Hof, C. (2010). Die Zeit der (erziehungswissenschaftlichen) Biographieforschung. Theoretische, methodologische und empirische Aspekte ihrer Fortschreibung. In J. Ecarius \& B. Schäffer (Hrsg.), Typenbildung und Theoriegenerierung: Methoden und Methodologien qualitativer Bildungs- und Biographieforschung (S. 145-167). Opladen: Verlag Barbara Budrich.

Kade, J., \& Nolda, S. (2014a). 1984/2009- Bildungsbiografische Gegenwarten im Wandel von Kontextkonstellationen. Zeitschrift für Pädagogik, 4, 589-606. 
Kade, J., \& Nolda, S. (2014b). Individualitätsperformanz. Bildungsbiographische Anspruchsindividualitäten in sich wandelnden Kontexten. In A. Geimer \& F. Rosenberg, von (Hrsg.), Kulturelle Differenzen (S. 125-154). Wiesbaden: VS Verlag.

Koller, H.-C. (2012). Bildung anders denken: Einführung in die Theorie transformatorischer Bildungsprozesse. Stuttgart: Kohlhammer.

Kruse, A. (1987). Biographische Methode und Exploration. In G. Jüttemann \& H. Thomae (Hrsg.), Biographie und Psychologie (S. 119-137). Berlin: Springer.

Langewand, A. (2004). Bildung. In D. Lenzen (Hrsg.), Erziehungswissenschaft: Ein Grundkurs (S. 69-98). Reinbek: Rowohlt.

Leipold, B. (2012). Lebenslanges Lernen und Bildung im Alter. Stuttgart: Kohlhammer.

Lessenich, S. (2009). Lohn und Leistung, Schuld und Verantwortung: Das Alter in der Aktivgesellschaft. In S. van Dyck \& S. Lessenich (Hrsg.), Die ,jungen Alten “ zwischen Aktivität und Widerstand (S. 279-295). Frankfurt a. M.: Campus.

Lessenich, S. (2014). Einfach nicht totzukriegen. Das Alter in der ,alternden Gesellschaft“. Merkur. Deutsche Zeitschrift für europäisches Denken, 5, 454-460.

Martin, P., Ettrich, K. U., Lehr, U., Roether, D., Martin, M., \& Fischer-Cyrulies, A. (Hrsg.). (2000). Aspekte der Entwicklung im mittleren und höheren Lebensalter: Ergebnisse der Interdisziplinären Längsschnittstudie des Erwachsenenalters (ILSE). Darmstadt: Steinkopff.

Nittel, D. (1999). Erziehungswissenschaften/Pädagogik. In B. Jansen, F. Karl, H. Radebold, \& R. SchmitzScherzer (Hrsg.), Soziale Gerontologie: Ein Handbuch für Lehre und Praxis (S. 356-369). Weinheim: Beltz.

Schäffer, B. (2012). Generation. Eine Analysekategorie für die Erwachsenen- und Weiterbildungsforschung. In B. Schäffer \& O. Dörner (Hrsg.), Handbuch Qualitative Erwachsenen- und Weiterbildungsforschung (S. 474-489). Opladen: Verlag Barbara Budrich.

Thomae, H. (1987). Psychologische Biographik als Synthese idiographischer und nomothetischer Forschung. In G. Jüttemann \& H. Thomae (Hrsg.), Biographie und Psychologie (S. 108-116). Berlin: Springer Verlag.

Thomae, H. (1996). Das Individuum und seine Welt. Eine Persönlichkeitstheorie. Göttingen: Hogrefe. 